\title{
Fuzzy maximum capacity and occupancy time rate measurements in urban railway lines
}

\author{
L. M. Navarro ${ }^{1 *}$, A. Fernandez-Cardador ${ }^{2}$ and A. P. Cucala ${ }^{2}$
}

\begin{abstract}
Nowadays, railway transport capacity is an important bottleneck for many railway operators that face its ever-increasing demand. This poses a challenge to existing lines as operation under conditions close to saturation tends be unstable. Capacity in urban railway systems depends largely on dwell times at platforms, but capacity measures proposed in literature rarely include the uncertainty associated to these times. In this paper this uncertainty is modeled as fuzzy numbers and two new capacity measures are proposed: the Fuzzy Maximum Capacity and the Fuzzy Occupancy Time Rate. The proposed model makes use of a railway simulator that enables route compression to obtain the conflict-free compressed time of the section under study.

Three practical capacity problems from the perspective of the railway traffic operator have been presented and solved. The new measures provide more information to the railway operator than the standard UIC method that does not include uncertainty regarding dwell times. Finally, the model has been applied to the section Gràcia-Sarrià, belonging to the Spanish railway operator FGC.
\end{abstract}

Keywords: Fuzzy, Urban transport, Dwell times, Capacity

\section{Introduction}

Nowadays, many of the urban railway lines are severely congested, especially during rush hour. In 1950, only $30 \%$ of the population lived in cities while at the present time this figure has increased to $54 \%$ and it is expected to reach $66 \%$ by 2050 [30]. Operators and managers of railway infrastructures are facing the major challenge of increasing the available capacity at existing installations to cover the expected growth of demand while, at the same time, coping with the operation of installations under conditions close to saturation which by nature are prone to be unstable.

Transport capacity is a vital component of railway systems, defined by the amount of rolling stock per unit of time that the system will be able to move [6]. Even though the concept of capacity is intuitive, its technical quantification in order to make it useful in the evaluation and proper design of railway infrastructures is

* Correspondence: luismiguel.navarro@siemens.com

${ }^{1}$ Siemens Rail Automation S.A.U, Cornellà de Llobregat, Spain

Full list of author information is available at the end of the article complex. This has led to the definition of different types of capacity in the literature $[1,15,16,21]$.

- Theoretical or maximum capacity: Maximum volume of traffic (usually expressed as number of trains per hour) that can travel through a railway network or network section assuming that the circulation of trains is not affected by signaling systems.

- Practical capacity: Volume of traffic that can travel through the system permanently maintaining a reasonable quality of service. It is a value smaller than theoretical capacity, because buffer time margins between trains are required to avoid small delays being transmitted to other trains [6, 11, 24].

- Operative Capacity used by a timetable: it is the volume of traffic contained in a timetable for a period of time.

- Available capacity: difference between the practical capacity and the operative capacity. It offers information about the residual capacity of a timetable.

Transport capacity depends on multiple factors, [1, 6, 27, 28], such as: 
- Infrastructure design: Track topology, gradients and speed limitations.

- Trains performance that limit the safety distance allowed between two consecutive trains.

- Signaling systems: They are in charge of guaranteeing safe train movements.

- Traffic, which is defined as the mix of trains, train sequences and priority rules at railway nodes. Capacity is reduced when trains with different speeds or lengths travel one after another, or under a different dwelling schedule [23].

- Dwell times at stations have a great influence on capacity. They are affected by door opening and closing times as well as time waiting to depart once the doors close, passenger flow time and time the doors remain open after passenger flow ceases [14].

- Reversing routes at terminus stations, where trains change direction.

As regards capacity calculation, there are analytical methods available such as the one proposed by the UIC (International Union of Railways) in its 405 leaflet [26], that allows obtaining a theoretical capacity value in a relatively simple manner by using mathematical expressions applied to simplified models of the system. These methods are based on real-world data of infrastructure and timetables, but they are only useful as a starting point or to detect capacity bottlenecks.

On the other hand, there are available methods based on timetable compression. UIC proposes in [29] their reference method of capacity calculation (UIC 406R). It aims to establish an international standard to evaluate capacity in different environments, by establishing a common framework and uniform principles.

The compression method eliminates buffer times between trains, calculating the maximum capacity as the number of trains contained within the compressed time (number of trains per hour). The occupancy time rate measure of a given timetable is defined as the compressed time divided by the timetable time window. This value can be compared to the reference values proposed by the UIC for various types of railway lines (Table 1).

In suburban lines this capacity calculation is heavily dependent on dwelling times, considering that they suppose an important percentage of the compressed time (for example, in Vallès line of the Catalan operator FGC they suppose up to $20 \%$ during peak hours).

Table 1 Proposed occupancy time rate values

\begin{tabular}{lll}
\hline Type of line & Peak Hour & Daily Period \\
\hline Dedicated suburban passenger traffic & $85 \%$ & $70 \%$ \\
Dedicated high-speed line & $75 \%$ & $60 \%$ \\
Mixed-traffic lines & $75 \%$ & $60 \%$ \\
\hline
\end{tabular}

An effective procedure to calculate the compressed time and the maximum capacity is the use of simulation models that allow reproducing the full railway system at a micro level (using tools like OpenTrack or MultiRail). This is accomplished by approximating the trains until they could not get closer without affecting each other (conflict-free compressed time). Some works present their own simulation tool $[2,10,17]$. Goverde et al [10] calculates capacity using the compression method. It considers uncertainty by means of Weibull distributions line entry times, but dwelling times are considered fixed.

The major part of performed works about capacity does not take into account uncertainty related to dwelling times, although there are some exceptions. In [18], dwell time variability is considered by gathering real-world data to calculate the conflict probability in a timetable. A revision of train dwell time models is performed in [25]. They all gather real-world data from several installations, the work concludes that the amount of passengers getting on and off and waiting at platforms are the most influencing parameters. Some of the works in the literature are completely centered on the study of passengers influence on dwelling times $[4,13,22,32]$.

In some situations there are not real-world data available, this is the case of greenfield projects where no previous information is available or when the timetables of a line need to be modified. In both cases, the previous statistical data of the line are no longer valid. In this situation, an approach based on fuzzy modeling proves to be especially convenient to include uncertainty in the model.

In [5] delays at the departure station are modeled using fuzzy numbers in order to optimize the timetable of a train subject to punctuality restrictions. Milinković et al [19] proposes to use expert knowledge to define rules and fuzzy sets to calculate primary and secondary delays based on a fuzzy Petri net. In [31] a model is proposed to solve the timetable planning of a line by applying fuzzy information on passenger demand and [12] shows a model to obtain the best trains timetable by using fuzzy AHP. In [9] a dispatching support system for railway operation control is described, it uses Petri Nets and Fuzzy Sets to model rule-based expert knowledge. In the previous works the use of fuzzy numbers has proven its utility to model uncertainty in several situations. However, this approach has not yet been applied to capacity calculation in a greenfield project, a redesign of an existing infrastructure or a timetable modification.

In the present work a fuzzy capacity model for railway lines is proposed. Uncertainty in dwell times is introduced by using fuzzy numbers. As a result, new measures of fuzzy maximum capacity and fuzzy occupancy time rate are proposed. The proposed method provides more information to the railway operator than the standard UIC method that does not include uncertainty 
regarding dwell times. The model permits as well adjusting the level of fulfillment of the UIC time margins to calculate the operative capacity.

Fuzzy capacity is compared with the operative capacity of a timetable, evaluating if the timetable is feasible under the current signaling system design. Fuzzy occupancy time rate is used to calculate the degree of compliance to the UIC robustness reference values. In addition, the model allows calculating the maximum operative capacity that achieves the UIC robustness requirement with a given level of possibility or necessity as a target value.

This approach is considered especially useful in urban railway lines with frequent stops and equipped with automatic driving systems, where the main source of uncertainty is located in dwell times due to passengers getting on and off, and not in running times [7].

The proposed model is applied to a real case in a section of the Vallès line of the Catalan operator FGC, which is currently saturated and under study to be partially redesigned. A railway traffic simulator based on OpenTrack has been configured and used to apply the UIC timetable compression method. The fuzzy maximum capacity model and the fuzzy occupancy rate are presented in the second section, including the model description, the proposed applications of the model and the model resolution. In the third section the case study is presented. Finally, the work conclusions are presented in section 4 .

\section{Fuzzy capacity model}

\subsection{Model description}

In this section, a model to calculate maximum capacity and occupancy time rate measurements using fuzzy numbers is proposed. This allows taking into account uncertainty regarding dwell times at platforms in urban railway lines and analyzing capacity dependency in relation to those times. To that effect, the compression method defined in [29] is applied. The compression method is a generalized method for calculating capacity consumption section by section. An example of its application is shown in Fig. 1; the left figure shows 11 consecutive train paths departing from Gràcia station with destination Sarrià. The $\mathrm{T}_{\text {ope }}$ is reflecting the cycle time of 2 trains following a certain timetable.

The right figure shows the same train paths but compressed until trains cannot get any closer without affecting each other, therefore eliminating buffer time margins between trains.

Given a periodic traffic pattern that determines the sequence of trains in the cycle and a signaling and operation system the maximum line capacity is calculated as:

$$
\mathrm{C}=\frac{3600 \cdot n}{T}
$$

Where $\mathrm{T}$ (in seconds) is the conflict-free compressed time of the traffic pattern, according to UIC, measured in seconds and $\mathrm{n}$ is the number of trains circulating in the pattern. Therefore, the capacity $\mathrm{C}$ is measured in
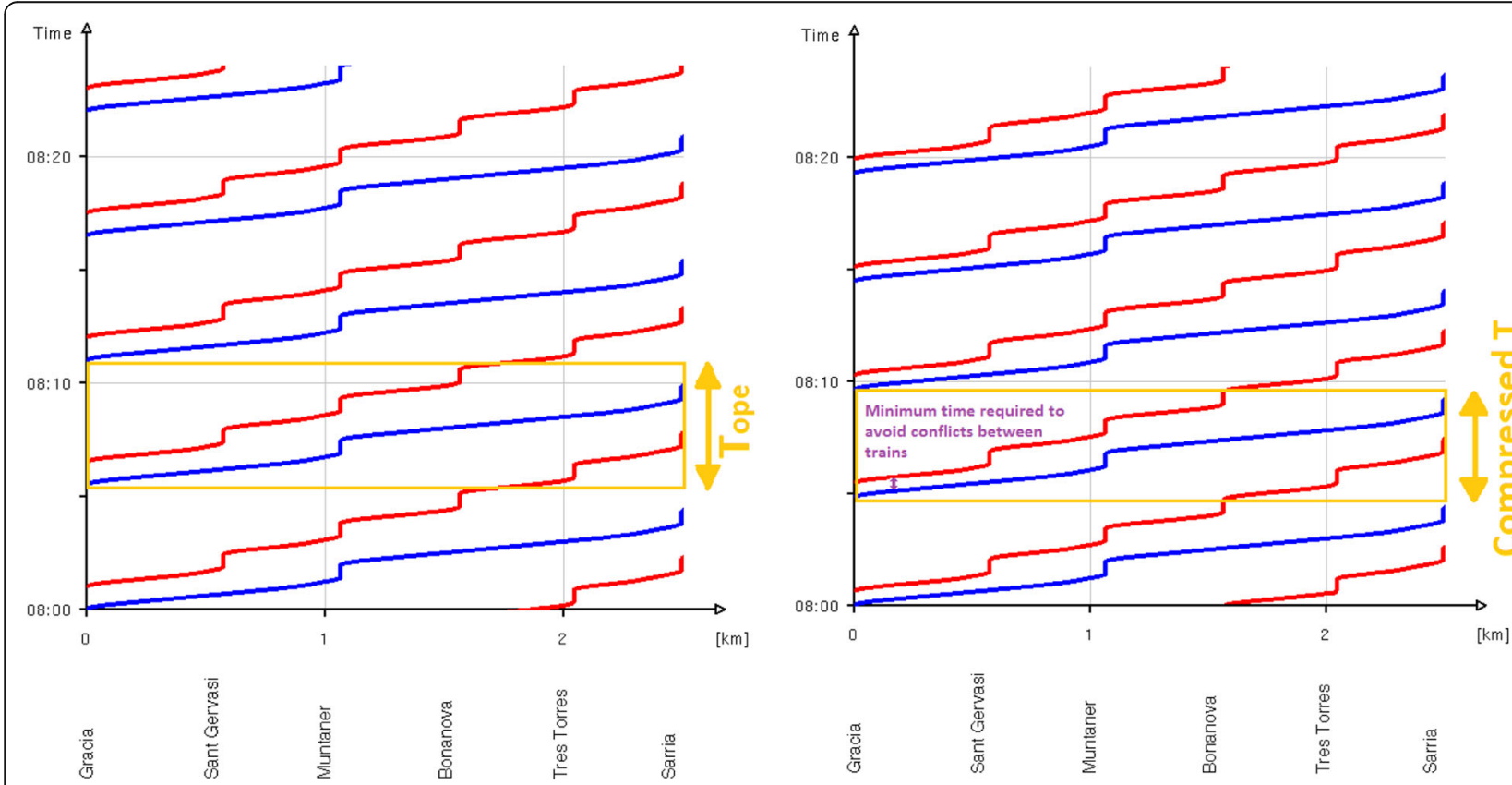

Fig. 1 Train paths diagram and the timetable compression method (Left: Uncompressed paths from the schedule. Tope reflects the periodic cycle time of 2 trains, Right: Compressed paths) 
trains per hour. After setting the traffic pattern, the compressed time $\mathrm{T}$ can be expressed as a function of dwell times in the stations of the analyzed line section:

$$
\mathrm{T}=\mathrm{f}\left(T_{D k}\right) \text { Where } \mathrm{k}=1,2 \ldots \mathrm{S}
$$

Where $T_{D k}$ is the dwell time at each platform $\mathrm{k}$, and $\mathrm{S}$ is the number of stops. Function $f$ will be calculated in the case study by using a detailed traffic, signaling and control systems simulator.

$$
\mathrm{C}=\frac{3600 \cdot n}{\mathrm{f}\left(T_{D k}\right)}
$$

The uncertainty associated to dwell times can be modeled as fuzzy numbers, and thus, the obtained compressed time is as well a fuzzy number:

$$
\tilde{\mathrm{T}}=\mathrm{f}\left(\tilde{T}_{D k}\right) \text { Where } \mathrm{K}=1,2 \ldots \mathrm{S}
$$

In this way, the proposed Fuzzy Maximum Capacity (see Eq. 5) is a function of the fuzzy dwell times:

$$
\tilde{\mathrm{C}}=\frac{3600 \cdot n}{\mathrm{f}\left(\tilde{T}_{D k}\right)}=F\left(\tilde{T}_{D K}\right)
$$

The function $f$ increases with Dwell Times. Hence, the maximum capacity decreases with those times. Thus, it is possible to easily calculate the fuzzy maximum capacity $\tilde{C}$ by means of $\alpha$-cut arithmetic [3].

The upper limit of each $\alpha$-cut of the fuzzy $\tilde{C}\left(C^{\bar{\alpha}}\right)$ can be obtained considering the lower limit of the $\alpha$-cut of the fuzzy $\tilde{\mathrm{T}}_{\mathrm{Dk}}\left(\mathrm{Td}^{\alpha_{-}}\right)$(see Eq. 6 and Fig. 1). Similarly, the lower limit of each $\tilde{C} \alpha$-cut $\left(C^{\alpha_{-}}\right)$can be obtained using the upper limit of the $\tilde{\mathrm{T}}_{\mathrm{Dk}} \alpha$-cut ( $\mathrm{Td}^{\bar{\alpha}}$ ) (see Eq.7 and Fig. 2). This way, by varying the $\alpha$ value between 0 and 1 , the whole $\tilde{C}$ set can be obtained.

$$
\mathrm{C}^{\bar{\alpha}}=\mathrm{F}\left(\mathrm{Td}^{\underline{\alpha}}\right)
$$

$$
\mathrm{C}^{\alpha_{-}}=\mathrm{F}\left(\mathrm{Td}^{\bar{\alpha}}\right)
$$

Given the fuzzy maximum capacity, and a timetable with an associated operative capacity $\mathrm{C}_{\mathrm{ope}}$, the Fuzzy Occupancy Time Rate Ũ can be defined as:

$$
\tilde{\mathrm{U}}=\frac{C_{\text {ope }}}{\tilde{\mathrm{C}}} \cdot 100
$$

\subsection{Railway traffic operator applications}

Using the previous model definitions of $\tilde{C}$ and $\tilde{U}$, three practical problems from the perspective of the railway traffic operator could be set out:

- Is the operative capacity achievable?

- Does the operative capacity keep enough reliability margins?

- Calculation of the highest $\mathrm{C}_{\text {ope }}$ that fulfills the recommended UIC Occupancy Time margins with a target possibility/necessity value

These three applications are described and solved in the following subsections.

\subsubsection{First application: is the operative capacity achievable?} Given a timetable, the first question is to determine if the associated operative capacity $\mathrm{C}_{\text {ope }}$ is below the Fuzzy Maximum Capacity, to asses if the timetable is feasible under the current signaling system (see Eq. 9):

$$
C_{\text {ope }} \leq \tilde{\mathrm{C}}
$$

The advantage of using the fuzzy value comparison instead of the crisp one is that the inherent uncertainty of dwell times is properly taken into account. The fuzzy number $\tilde{C}$ is compared to the crisp number $\mathrm{C}_{\text {ope }}$ in terms of possibility $\Pi$ and necessity $\mathrm{N}$ measures [8], providing the possibility and the necessity that the $\mathrm{C}_{\text {ope }}$ is below the fuzzy maximum capacity (Eqs. 10 and 11). Thus, Railway operators obtain more information about the degree of fulfillment of the maximum capacity.

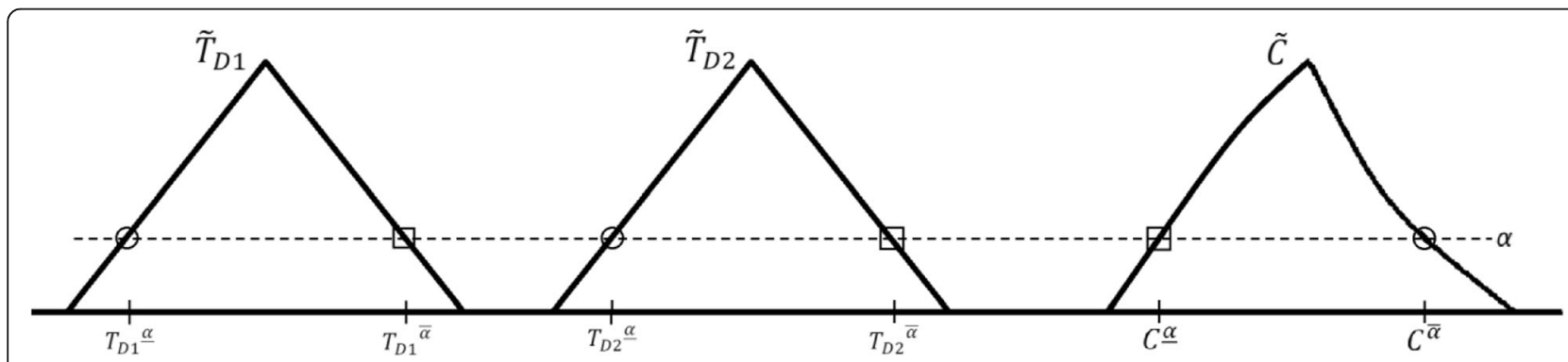

Fig. 2 Fuzzy capacity obtained from fuzzy Dwell Times 


$$
\begin{aligned}
& \Pi\left(\tilde{\mathrm{C}} \geq C_{\text {ope }}\right) \\
& \mathrm{N}\left(\tilde{\mathrm{C}} \geq C_{\text {ope }}\right)=1-\Pi\left(\tilde{\mathrm{C}}<C_{\text {ope }}\right)
\end{aligned}
$$

Two examples are presented below:

In Fig. 3 , the possibility of $\tilde{C}$ being greater than or equal to $\mathrm{C}_{\text {ope }}$ is $\alpha$, and the necessity of $\tilde{C}$ being greater than or equal to $\mathrm{C}_{\text {ope }}$ is zero, since:

$$
\mathrm{N}\left(\tilde{\mathrm{C}} \geq C_{\text {ope }}\right)=1-\Pi\left(\tilde{\mathrm{C}}<C_{\text {ope }}\right)=1-1=0
$$

In Fig. 4, the possibility of $\tilde{C}$ being greater than or

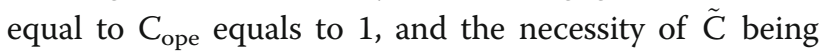
greater than or equal to $C_{\text {ope }}$ equals to $1-\alpha$, since:

$$
\mathrm{N}\left(\tilde{\mathrm{C}} \geq C_{\text {ope }}\right)=1-\Pi\left(\tilde{\mathrm{C}}<C_{\text {ope }}\right)=1-\alpha
$$

\subsubsection{Second application: does the operative capacity keep enough reliability margins?}

In the first application it has been checked if the Operative Capacity is less than the Theoretical fuzzy maximum Capacity. But this condition does not ensure that a timetable is robust enough to incidents. To conclude that a timetable allows an operation that is reliable (it can compensate delays by using enough time margins), the timetable needs to preserve certain occupancy time rate reference values [29]. The fuzzy occupancy time rate is calculated for a given timetable, as its associated operative capacity divided by the fuzzy maximum capacity (in \%) (Eq. 8).

Therefore, the Fuzzy Occupancy Time Rate of the line can be compared to the reference values proposed by the UIC:

$$
\tilde{\mathrm{U}} \leq U_{U I C}
$$

$$
\begin{aligned}
& \frac{\mathrm{C}_{\text {ope }}}{\tilde{\mathrm{C}}} 100 \leq \mathrm{U}_{\mathrm{UIC}} \\
& \frac{\mathrm{C}_{\text {ope }}}{\mathrm{U}_{\mathrm{UIC}}} 100 \leq \tilde{\mathrm{C}}
\end{aligned}
$$

Where $\mathrm{U}_{\mathrm{UIC}}$ is the maximum occupancy time rate reference value recommended by the UIC. This value is defined for every type of railway line and service hour (Table 1).

The degree of compliance to the UIC robustness requisite is evaluated again in terms of possibility and necessity measures, but this time comparing the fuzzy maximum capacity $\tilde{C}$ to the proportion of the operative capacity $C_{\text {ope }}$ over the UIC reference value $\mathrm{U}_{\mathrm{UIC}}$ (Eq. 16). Again, railway operators obtain information about the degree of fulfillment of the UIC recommended occupancy time rate values. Then, possibility and necessity measures associated with the fuzzy comparison are calculated with a procedure akin to the one described previously in the first application.

\subsubsection{Third application: calculation of the highest $C_{\text {ope }}$ that fulfills the recommended UIC occupancy time margins with a target possibility/necessity value}

Finally, once the fuzzy maximum capacity associated with an infrastructure and a certain traffic pattern has been calculated (taking into account the uncertainty in dwell times), the proposed model allows calculating the maximum operative capacity $\mathrm{C}_{\text {ope }}$ that achieves the UIC robustness requirement with a given level of possibility or necessity as a target. To that effect, the $C_{\text {ope }}$ of the following equations has to be calculated depending if the imposed requirement is a possibility value $\alpha_{\text {obj }}$ of a necessity value $\mathrm{N}_{\mathrm{obj}}$ :

$$
\prod\left(\frac{\mathrm{C}_{\text {ope }}}{\mathrm{U}_{\mathrm{UIC}}} 100 \leq \tilde{\mathrm{C}}\right)=\alpha_{\mathrm{obj}}
$$

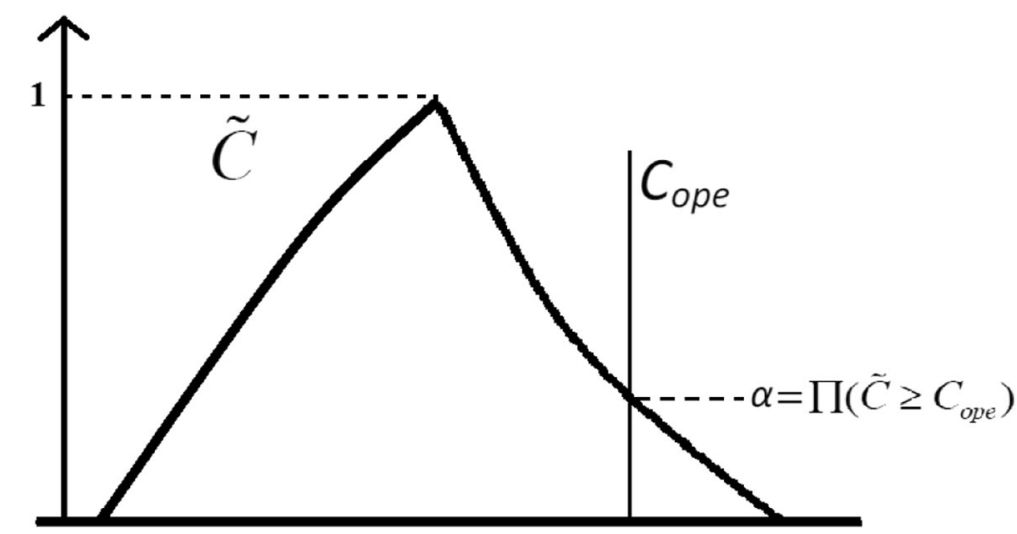

Fig. 3 Fuzzy maximum capacity compared to Operative Capacity $C_{o p e}$ at the right side 


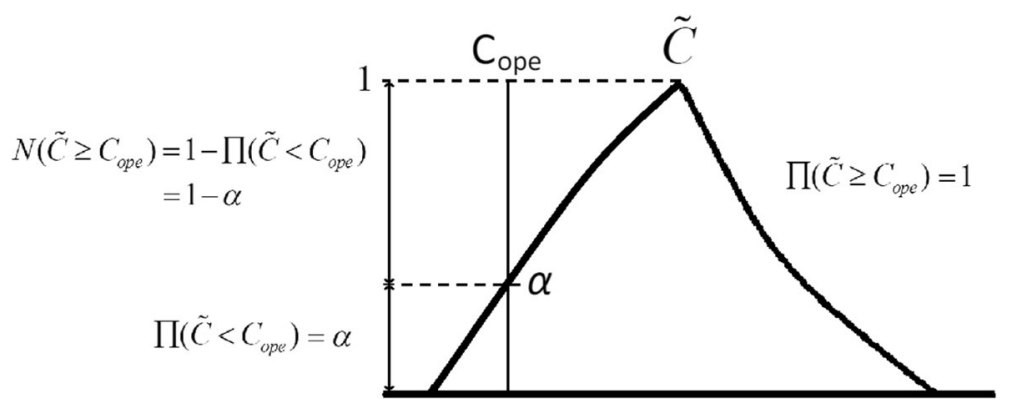

Fig. 4 Fuzzy maximum capacity compared to Operative Capacity, $C_{\text {ope }}$ at the left side

$$
\mathrm{N}\left(\frac{\mathrm{C}_{\text {ope }}}{\mathrm{U}_{\text {UIC }}} 100 \leq \tilde{\mathrm{C}}\right)=\mathrm{N}_{\text {obj }}
$$

Where $\alpha_{\mathrm{obj}}$ and $\mathrm{N}_{\mathrm{obj}}$ are the target levels of possibility and necessity that could be imposed as the level of fulfillment of the UIC recommended value.

\subsection{Model resolution}

In this section the procedure to solve the problems laid out in the previous section is described.

\subsubsection{Problems 1 (and 2) resolution}

A procedure to solve problems 1 and 2 without the need of generating the whole $\tilde{C}$ number, which would require repeated iterations, is proposed. This procedure is based on the alpha-cuts arithmetic (see Fig. 2). The method for the first problem is the following:

1. For $\alpha$ equal to 0 , the simulation function $F\left(T_{D k}\right)$ is applied using the $\mathrm{T}_{\mathrm{Dk}}{ }^{\alpha}{ }_{-}$values. The resulting capacity value is the upper limit of the $\alpha$-cut of $C^{\bar{\alpha}}$ with $\alpha$ equal to 0 (see Fig. 5). If this $C^{\bar{\alpha}}$ value is lower than $\mathrm{C}_{\text {ope }}$, the possibility and necessity of $\mathrm{C}_{\text {ope }} \leq \tilde{\mathrm{C}}$ are null and the algorithm ends.

2. For $\alpha$ equal to 0 , the simulation function $F\left(T_{D k}\right)$ is applied using the $\mathrm{T}_{\mathrm{Dk}}{ }^{\bar{\alpha}}$ values. The resulting capacity value is the lower limit of the $\alpha$-cut of $C^{\alpha}$ with $\alpha$ equal to 0 (see Fig. 5). If this $C^{\alpha}$ value is higher than $\mathrm{C}_{\text {ope }}$, the possibility and necessity of $\mathrm{C}_{\text {ope }} \leq \tilde{\mathrm{C}}$ are equal to 1 and the algorithm ends.

3. For $\alpha$ equal to 1 , the simulation function $F\left(T_{D k}\right)$ is applied using the $\mathrm{T}_{\mathrm{Dk}} \bar{\alpha}=\mathrm{T}_{\mathrm{Dk}} \underline{\alpha}$ values, that is, the core of the triangular fuzzy numbers. The resulting capacity value is the core of the fuzzy maximum capacity ( $\alpha$ equal to 1 ) (see Fig. 5 ).

4. If $C_{\text {ope }}$ is higher than $C^{\bar{\alpha}}=C^{\underline{\alpha}}$ with $\alpha$ equal to 1 , then the cutting point between $C_{\text {ope }}$ and $\tilde{C}$ has to be found in the upper limits of the $\alpha$-cuts of $\tilde{C}$ by using the bipartition method simulating $F\left(T_{D k}\right)$ with the lower limits of $\tilde{\mathrm{T}}_{\mathrm{Dk}} \alpha$-cuts. In this situation, the necessity value is null and the possibility value is calculated as the $\alpha$ value of the cutting point (see Fig. 3), and the algorithm ends.

5. If $C_{\text {ope }}$ is lower than $C^{\bar{\alpha}}=C^{\alpha}$ with $\alpha$ equal to 1 , then the cutting point between $\mathrm{C}_{\text {ope }}$ and $\tilde{C}$ has to be found in the lower limits of the $\alpha$-cuts of $\tilde{C}$ by using the bipartition method simulating $\mathrm{F}\left(\mathrm{T}_{\mathrm{Dk}}\right)$ with

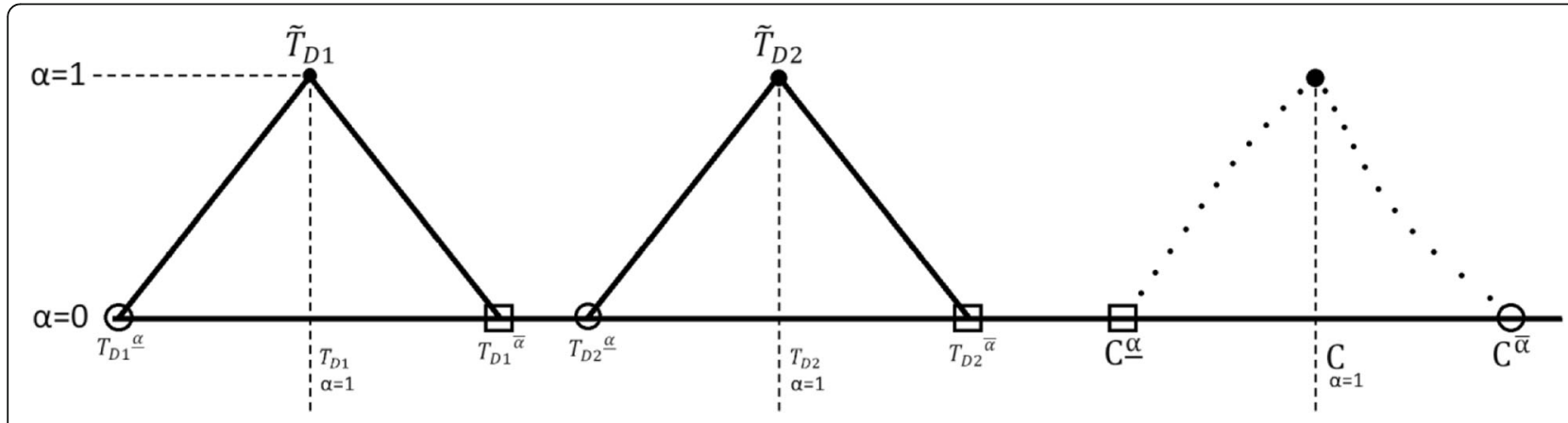

Fig. 5 Calculation of the core and the support of fuzzy maximum capacity obtained from triangular fuzzy Dwell Times, a-cuts method 
the upper limits of $\tilde{\mathrm{T}}_{\mathrm{Dk}} \alpha$-cuts. In this situation, the possibility value equals to 1 and the necessity value is calculated as 1 minus the $\alpha$ value in the cutting point (see Fig. 4), and the algorithm ends.

If the fuzzy $\tilde{\mathrm{T}}_{\mathrm{Dk}}$ numbers representing dwell times have a nucleus longer than 0 (for example trapezoidal fuzzy numbers), the same procedure can be applied.

The same procedure can be used to solve problem number 2 , considering that in problem 2 the fuzzy maximum capacity $\tilde{\mathrm{C}}$ has to be compared to $\frac{\mathrm{C}_{\text {ope }}}{\mathrm{U}_{\text {UIC }}} 100$ instead of $\mathrm{C}_{\text {ope }}$.

\subsubsection{Problem 3 resolution}

To calculate the limit $\mathrm{C}_{\text {ope }}$ of the timetable that fulfills the UIC reference value with a target possibility $\alpha_{\text {obj }}$ (thus, the associated necessity value is 0 ), $C_{\text {ope }}$ can be isolated out of the following equation:

$$
\prod\left(\frac{\mathrm{C}_{\mathrm{ope}}}{\mathrm{U}_{\mathrm{UIC}}} 100 \leq \tilde{\mathrm{C}}\right)=\alpha_{\mathrm{obj}}
$$

To this end, $C^{\bar{\alpha}}$ has to be calculated with $\alpha$ equal to $\alpha_{\text {obj }}$ (see Fig. 3), that is, just one simulation is required $F$ $\left(T_{D k}{ }^{\alpha}\right)$ with $\alpha$ equal to $\alpha_{\text {obj. }}$. Finally, the $C_{\text {ope }}$ that meets the specified requirement is obtained as the result of multiplying $C^{\overline{\alpha_{o b j}}}$ by $\mathrm{U}_{\mathrm{UIC}} / 100$.

If the accomplishment of a certain level of necessity $\mathrm{N}_{\text {obj }}$ is fixed as a goal (thus, the associated possibility value is 1$), C_{\text {ope }}$ can be calculated from the equation:

$$
\mathrm{N}\left(\frac{\mathrm{C}_{\text {ope }}}{\mathrm{U}_{\text {UIC }}} 100 \leq \tilde{\mathrm{C}}\right)=\mathrm{N}_{\text {obj }}
$$

For this purpose, $C^{\underline{\alpha}}$ has to be calculated (see Fig. 4) with $\alpha$ equal to 1 minus $\mathrm{N}_{\mathrm{obj}}$, that is, just one simulation is required $F\left(T d_{D k}{ }^{\bar{\alpha}}\right)$ with $\alpha$ equal to 1 minus $\mathrm{N}_{\text {obj}}$. Finally, the $\mathrm{C}_{\text {ope }}$ value that meets the specified requirement is obtained as the result of multiplying $C^{\underline{\alpha}}$ by $\mathrm{U}_{\mathrm{UIC}} / 100$.

\section{Case study}

A section of the FGC Barcelona-Vallès network has been chosen to apply the proposed model. FGC (Ferrocarrils de la Generalitat de Catalunya) is a railway company that operates several lines in Catalonia, a region located at the northeast of Spain.

The Barcelona-Vallès infrastructure (Fig. 6) is equipped with a safety system called ATP (Automatic Train Protection), which is in charge of supervising the train speed and applying the penalty brake in the event of safety conditions not being fulfilled. This line is equipped with a speed codes implementation scaled to $90 / 60 / 30 / 0 \mathrm{~km}$ per hour.

The section Gràcia-Sarrià covers one of the most congested parts of the network, comprising the nodes of Gràcia and Sarrià, which are two key points of the whole network. The first one is the main node serving several lines: L6, L7, S1, S2, S5 and S55.

With the aim of obtaining precise times of the itineraries in the installation, a parametric model of the section has been implemented using a simulation tool. This tool allows modelling line profile data and track topology as well as rolling stock and the routes for each interlocking of the line.

\subsection{Simulator description}

The infrastructure topology of the railway line has been developed in a graphical manner using the OpenTrack tool [20], which supports modelling a railway line by means of double vertex graphs (Fig. 7). Graphic elements have attributes that can be precisely configured by the user. Also, precise data of the rolling stock such as technical characteristics for every Electrical Multiple Unit, including traction efforts and speed diagrams, weight, length, adherence factor and power systems can

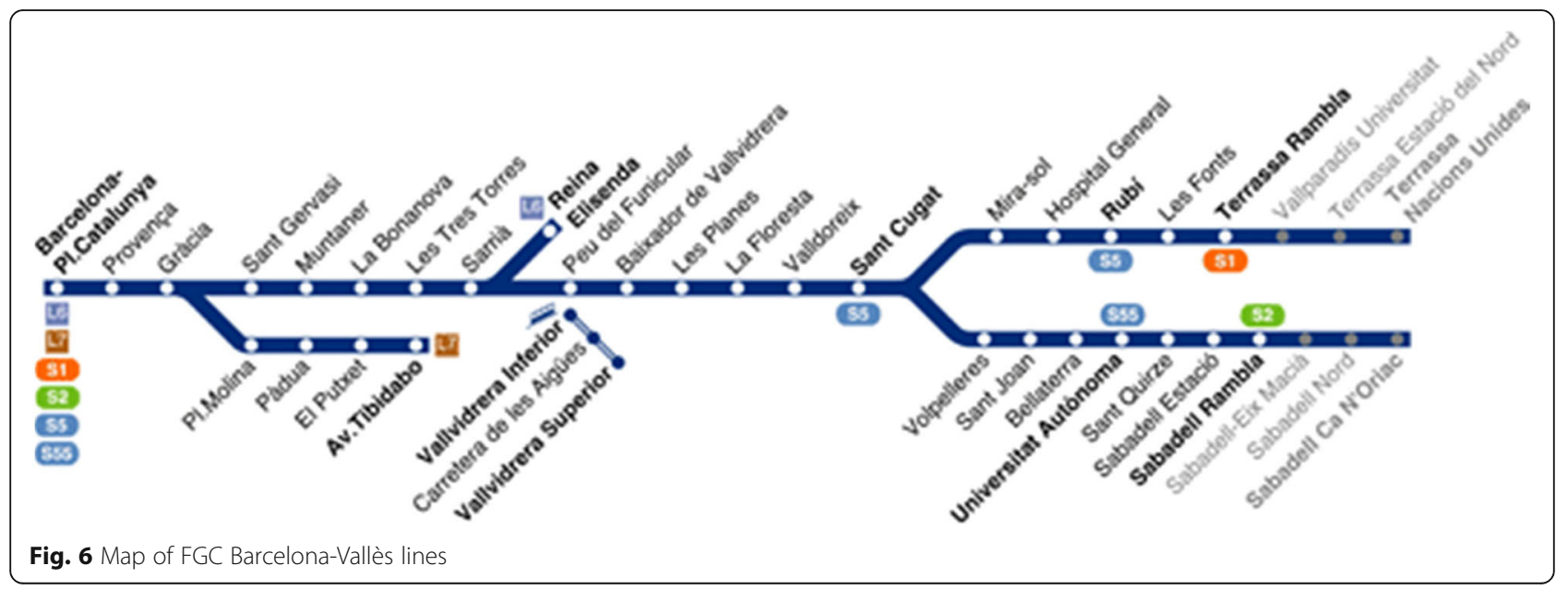




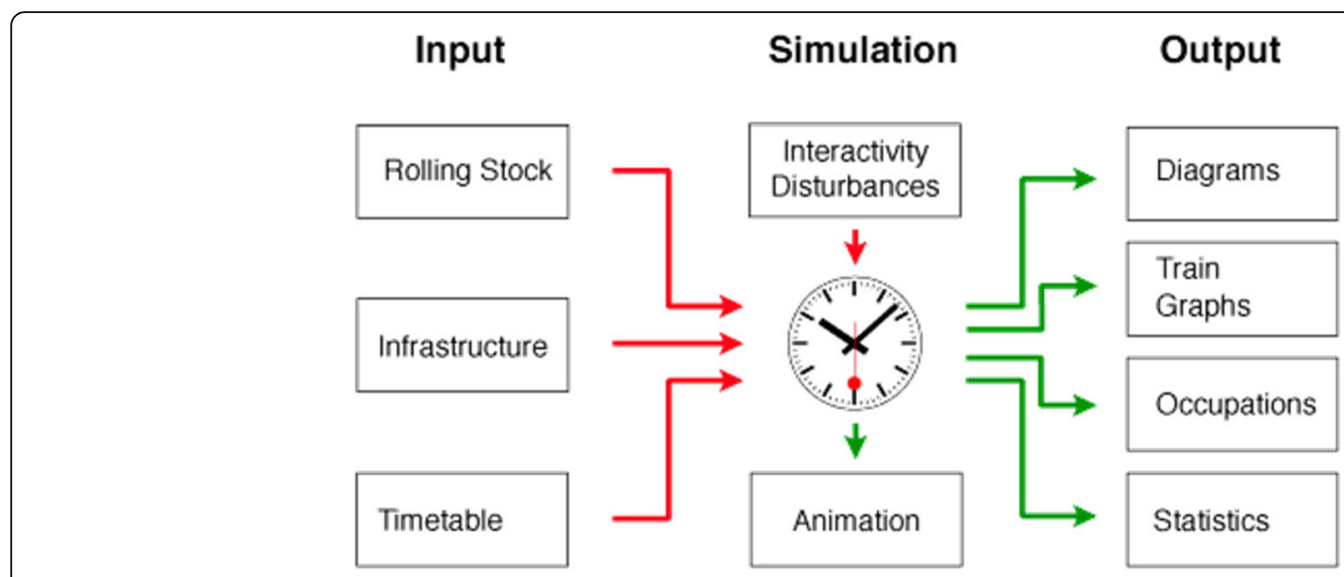

Fig. 7 Simulator operation process

be introduced. In particular, real data obtained from FGC series 112, 113 and 114 currently in service have been applied.

Signaling and ATP systems have been modeled using real data coming from the actual, such as track circuit lengths and gradients, signals, routes between signals, switches and ATP system features.

\subsection{Line section description}

The Gràcia-Sarrià section presents some conditions that make it particularly interesting for its analysis. It is an especially congested section, also Sarrià station has been remodeled recently with the aim of improving its capacity, and further improvements to the section are still required to be implemented. Therefore, it turns out to be a good testing ground to analyze its transport capacity.

\subsection{Description of the traffic pattern}

The simulated timetable of the line shows that in the line section from Gràcia to Sarrià, and from 8 to $9 \mathrm{~h}$ during weekdays, 21.8 trains are circulating in direction to Sarrià.

Those trains moving from Gràcia to Sarrià have a particular behavior, which is that half of them do not stop at three of the stations of the interval: Sant Gervasi, La Bonanova and Les Tres Torres. This makes the study of this section key when it comes to determining its Operative Capacity $\left(\mathrm{C}_{\text {ope }}\right)$. Table 2 shows the simulated timetable which illustrates the aforementioned characteristics.

The repeating cycle between Gràcia and Sarrià during the described part of the service is hence formed by 2 trains, one stopping at all 7 stations and another one stopping only at 4 of them.

The simulator is used to obtain the compressed time $\mathrm{T}$ (in seconds) of the traffic pattern, simulating the trains up to the limit where their circulations would be affected (UIC, 2004). Table 3 shows the main parameters of the compressed pattern simulated and its Time vs Distance diagram can be found in Fig. 1.

Figure 8 gives an example of the unhindered speed profile computed in the case study for the routes from Gràcia to Sarrià. This simulated speed profile has been contrasted with real data from the line and the results show its good accuracy.

\subsection{Triangular fuzzy dwell times}

To consider uncertainty in dwell times they have been modeled as triangular fuzzy numbers. The membership function is defined as follows, where $\mathrm{T}_{\mathrm{Dk}}$ is the core of the fuzzy dwell time (Eq. 21, Fig. 9):

$$
\tilde{T}_{D k}(\mathrm{x})=\left[\begin{array}{ccc}
o & \text { if } & x<T_{D k}{ }^{\alpha_{-}} \\
\frac{x-T_{D k} \alpha_{-}}{T_{D k}-T_{D k} \alpha_{-}} & \text {if } & T_{D k}{ }^{\alpha_{-} \leq x \leq T_{D k}} \\
\frac{T_{D k}{ }^{\bar{\alpha}}-x}{T_{D k}{ }^{\bar{\alpha}}-T_{D k}} & \text { if } & T_{D k} \leq x \leq T_{D k}{ }^{\bar{\alpha}} \\
0 & \text { if } & x>T_{D k}{ }^{\bar{\alpha}}
\end{array} \mid\right.
$$

Table 2 Simulated timetable of trains departing from Gràcia with destination Sarrià (08:00-09:00)

\begin{tabular}{llllll}
\hline Position & Line & Departure & Position & Line & Departure \\
\hline $1^{\text {st }}$ & S1 & $8: 00: 00$ & $12^{\text {th }}$ & L6 & $8: 28: 30$ \\
$2^{\text {nd }}$ & L6 & $8: 01: 00$ & $13^{\text {th }}$ & S1 & $8: 33: 00$ \\
$3^{\text {rd }}$ & S2 & $8: 05: 30$ & $14^{\text {th }}$ & L6 & $8: 34: 00$ \\
$4^{\text {th }}$ & $\mathrm{L} 6$ & $8: 06: 30$ & $15^{\text {th }}$ & S2 & $8: 38: 30$ \\
$5^{\text {th }}$ & S1 & $8: 11: 00$ & $16^{\text {th }}$ & L6 & $8: 39: 30$ \\
$6^{\text {th }}$ & L6 & $8: 12: 00$ & $17^{\text {th }}$ & S1 & $8: 44: 00$ \\
$7^{\text {th }}$ & $S 2$ & $8: 16: 30$ & $18^{\text {th }}$ & L6 & $8: 45: 00$ \\
$8^{\text {th }}$ & L6 & $8: 17: 30$ & $19^{\text {th }}$ & S2 & $8: 49: 30$ \\
$9^{\text {th }}$ & $S 1$ & $8: 22: 00$ & $20^{\text {th }}$ & L6 & $8: 50: 30$ \\
$10^{\text {th }}$ & L6 & $8: 23: 00$ & $21^{\text {st }}$ & S1 & $8: 55: 00$ \\
$11^{\text {th }}$ & $S 2$ & $8: 27: 30$ & $22^{\text {nd }}$ & L6 & $8: 56: 00$ \\
\hline
\end{tabular}


Table 3 Main parameters of the simulated compressed pattern

\begin{tabular}{ll}
\hline Parameter & Value \\
\hline Dwell Time at stations & $30 \mathrm{~s}$ \\
Compressed Cycle Time & $289 \mathrm{~s}$ \\
(2 trains, Sarrià direction) & \\
\hline
\end{tabular}

The three practical applications from the perspective of the traffic operator previously proposed in section 2.2 are analyzed. Also, a sensitivity analysis is presented with the objective of assessing the impact of variations in the core and support of the fuzzy dwell times.

Variations of the fuzzy dwell times $\mathrm{T}_{\mathrm{Dk}}$ core between 20 to $40 \mathrm{~s}$ are going to be considered. Likewise, $\mathrm{T}_{\mathrm{DL}}$ support variations between 15 and $25 \mathrm{~s}$ are taken into account as well.

3.5 First application: is the operative capacity achievable? The first step is to verify whether the operative capacity $\mathrm{C}_{\text {ope }}$, defined by the operator, is below the Fuzzy Maximum Capacity. According to the simulated timetable, this section of the line is operated with a $C_{\text {ope }}$ of 21.8 trains per hour during the rush hour of the daily service. The fuzzy maximum capacity shown in Fig. 10, has been obtained using a fuzzy dwell time with a $\mathrm{T}_{\mathrm{Dk}}$ of $30 \mathrm{~s}$ and a $\mathrm{T}_{\mathrm{DL}}$ of $25 \mathrm{~s}$, which could be typical values according to the experience of the line operator.

Under that scenario, the possibility and necessity measures of fulfilling the fuzzy constraint $\tilde{\mathrm{C}} \geq C_{\text {ope }}$ are calculated as:

$$
\begin{aligned}
& \mathrm{N}\left(\tilde{\mathrm{C}} \geq C_{\text {ope }}\right)=1-\Pi\left(\tilde{\mathrm{C}}<C_{\text {ope }}\right)=1-\alpha \\
& \mathrm{N}\left(\tilde{\mathrm{C}} \geq C_{\text {ope }}\right)=1-\alpha=0,83
\end{aligned}
$$

$$
\Pi\left(\tilde{\mathrm{C}} \geq C_{\text {ope }}\right)=1
$$

The results show that, even during the rush hour, the first requirement is accomplished with a high degree of certainty (although no reliability margin has been considered yet to face an incident without disrupting the service).

For the sake of comparison, by using the same input parameters but with crisp values (considering the core of the Fuzzy Dwell times) instead of fuzzy ones, the following maximum capacity is obtained:

$$
\mathrm{C}=\frac{3600 \cdot n}{T}=\frac{3600 \cdot 2}{289}=24,91 t / h
$$

The crisp value shows that the $\mathrm{C}_{\text {ope }}$ of 21.8 trains per hour would always be accomplished. Hence, taking uncertainty into account by means of fuzzy numbers provides the operator richer and more complete information than just using crisp values.

3.6 Second application: apart from being achievable, does the operative capacity keep enough reliability margins?

In a similar way to the first application, in the second one the operative capacity is compared considering the margin proposed by the UIC in terms of the occupancy time rate. The fuzzy occupancy time rate $\tilde{U}$ shown in Fig. 11, is obtained using a fuzzy dwell time with $\mathrm{T}_{\mathrm{Dk}}$ of $30 \mathrm{~s}$ and a $\mathrm{T}_{\mathrm{DL}}$ of $25 \mathrm{~s}$.

Given the reference occupancy time rate $\mathrm{U}_{\mathrm{UIC}}$ of $85 \%$ proposed by UIC, the possibility and necessity measures of fulfilling the fuzzy constraint $\tilde{U} \leq U_{U I C}$ are calculated as:

$$
\begin{aligned}
& \mathrm{N}\left(\tilde{\mathrm{U}} \leq U_{\text {UIC }}\right)=1-\Pi\left(\tilde{\mathrm{U}}>U_{\text {UIC }}\right)=1-1=0 \\
& \Pi\left(\tilde{\mathrm{U}} \leq U_{\text {UIC }}\right)=\alpha=0,78
\end{aligned}
$$

In light of the results, the second requirement can be accomplished with a degree of certainty during the

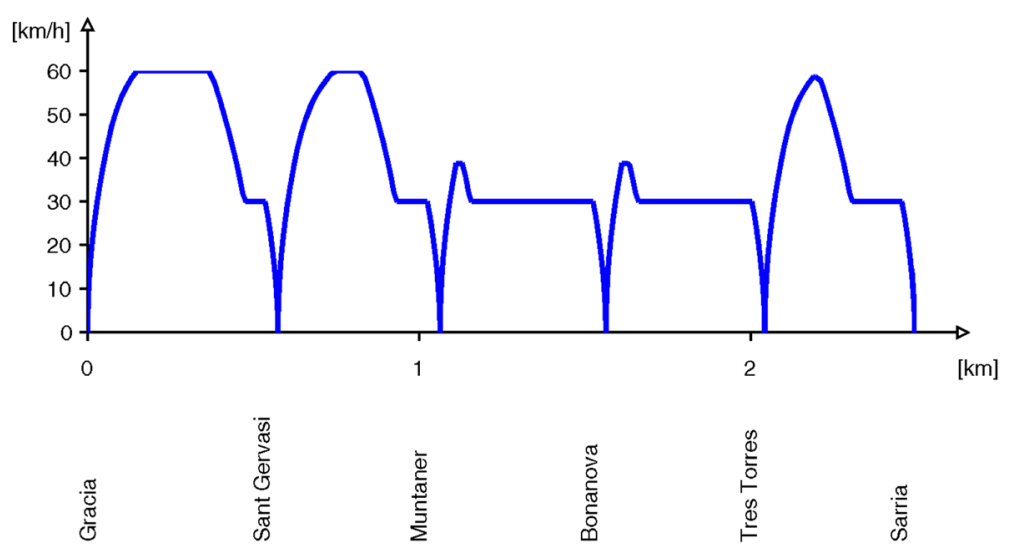

Fig. 8 Simulated speed profile of a train circulating from Gràcia to Sarrià using the ATP system. Train not hindered by other circulations and stopping at all stations 


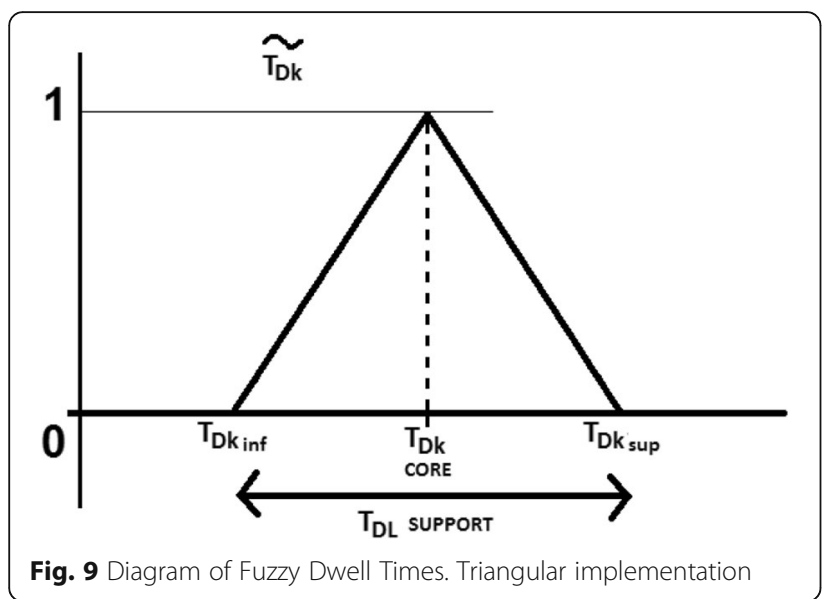

rush hour of operation. If the same calculation is performed using crisp values, the following results are obtained:

$$
\begin{aligned}
& \frac{C_{\text {ope }}}{\mathrm{C}} 100 \leq \mathrm{U}_{\text {UIC }} \quad(28) \\
& \frac{22 \mathrm{t} / \mathrm{h}}{24,91 \mathrm{t} / \mathrm{h}} 100=88,32 \%>85 \%
\end{aligned}
$$

In this case, the perception about the degree of certainty is lost entirely. Then, as a conclusion, it would seem that the operative capacity is not achievable by any means if the occupancy time rate value of $85 \%$ proposed by the UIC is taken into consideration.

3.7 Third application: calculation of the highest $C_{\text {ope }}$ that fulfills the recommended UIC occupancy time margins with a target possibility/necessity value

The values of possibility and necessity that fulfill the required UIC occupancy time rate value and $C_{\text {ope }}$ of the section at the same time can be found by calculating Eq. 30. The numerical values have been obtained by using a $\mathrm{T}_{\mathrm{Dk}}$ value of $30 \mathrm{~s}$ with a $\mathrm{T}_{\mathrm{DL}}$ of $25 \mathrm{~s}$, and $\alpha_{\mathrm{obj}}=0,5$ and $\mathrm{N}_{\text {obj }}=0$ :

$$
\begin{aligned}
& \prod\left(\frac{\mathrm{C}_{\text {ope }}}{\mathrm{U}_{\text {UIC }}} 100 \leq \tilde{\mathrm{C}}\right)=\alpha_{\text {obj }} \\
& \prod\left(\frac{\mathrm{C}_{\text {ope }}}{85 \%} 100 \leq \tilde{\mathrm{C}}\right)=0,5 \\
& \mathrm{C}_{\text {ope }}=23,18 \mathrm{t} / \mathrm{h}
\end{aligned}
$$

Therefore, under the dwell times defined and using the uncertainty parameters set previously, the highest $\mathrm{C}_{\text {ope }}$ that fulfils the target possibility is 23,18 trains per hour.

Another example on this same scenario could be obtained. If the operator imposes a higher level of certainty on the fulfillment of the UIC Occupancy Time margins, he would impose a necessity level instead (that is stricter than a possibility level). In the following example, $\mathrm{C}_{\text {ope }}$ is calculated by setting $\mathrm{N}_{\mathrm{obj}}=0,42$ and $\alpha_{\mathrm{obj}}=1$ :

$$
\begin{aligned}
& \mathrm{N}\left(\frac{\mathrm{C}_{\text {ope }}}{85 \%} 100 \leq \tilde{\mathrm{C}}\right)=0,42 \\
& \mathrm{C}_{\text {ope }}=19,75 \mathrm{t} / \mathrm{h}
\end{aligned}
$$

In this case, the calculated $C_{\text {ope }}$ is $19,75 \mathrm{t} / \mathrm{h}$. When a necessity level is imposed, the operative capacity calculated is lower than the one obtained when a possibility value is imposed.

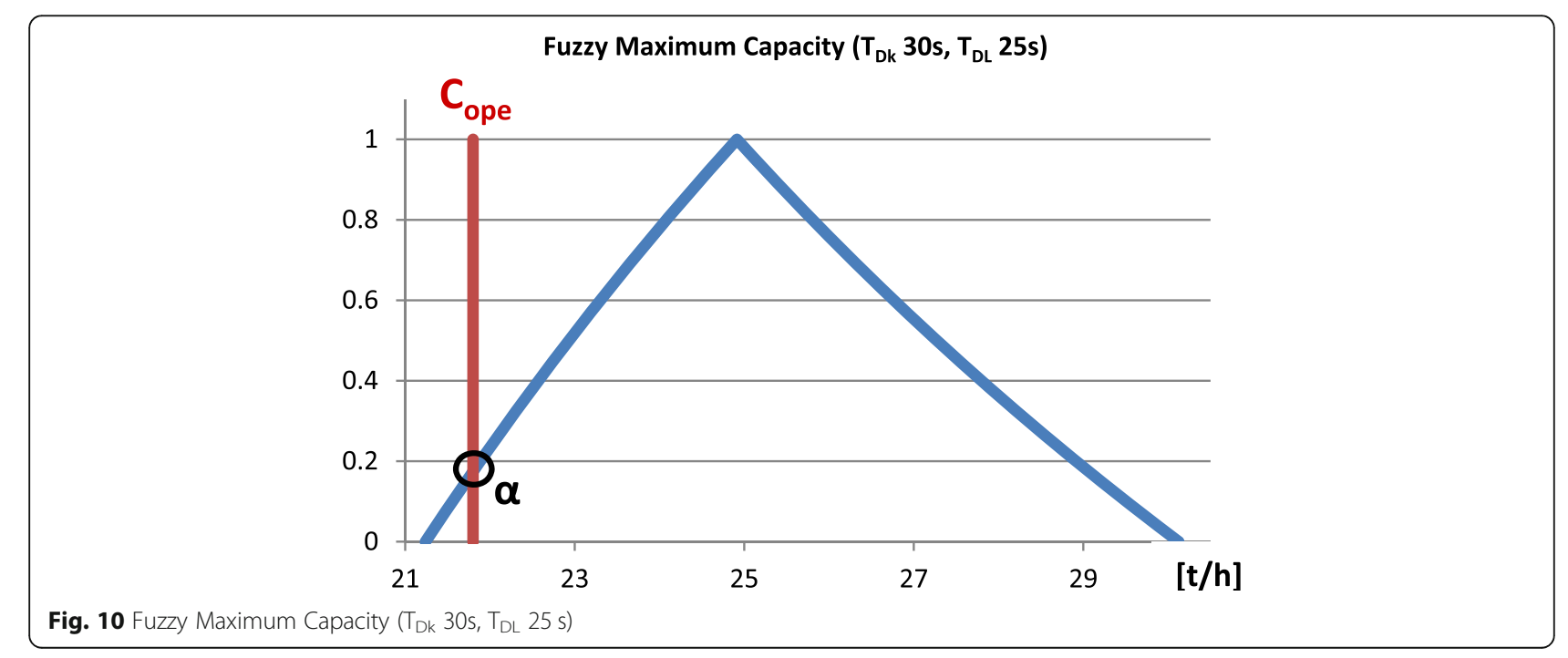


Fuzzy Occupancy Time Rate $\left(T_{D k} 30 s, T_{D L} 25 s\right)$

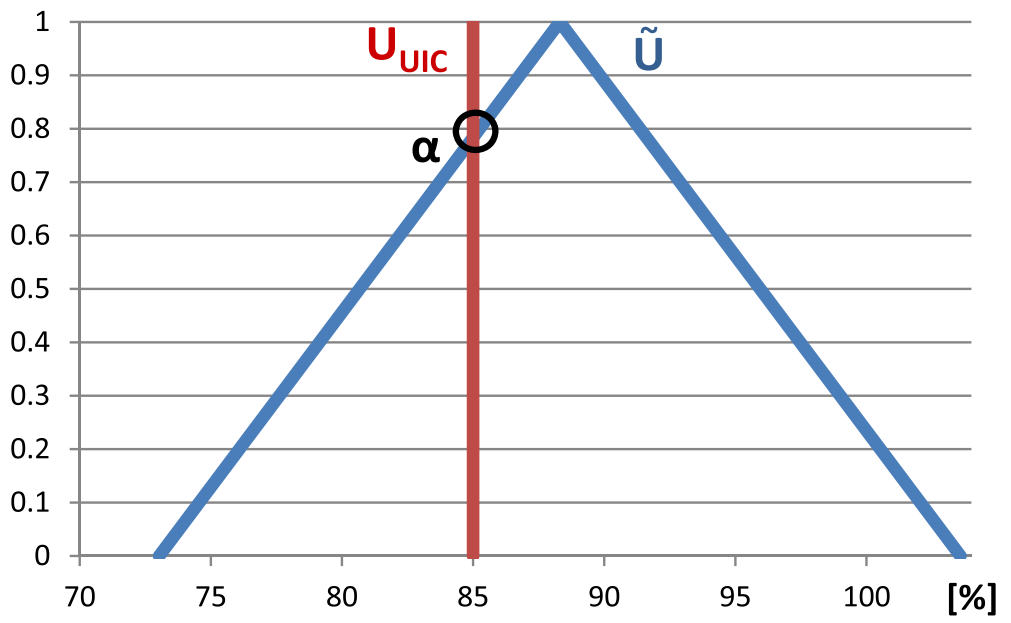

Fig. 11 Fuzzy Occupancy Time Rate ( $\left.T_{D k} 30 s, T_{D L} 25 s\right)$

\subsection{Sensitivity analysis}

This section is aimed to show a sensitivity analysis of the capacity considering different fuzzy dwell time possibility distributions. To this end, all fuzzy maximum capacities have been obtained for fuzzy dwell times with core $\mathrm{T}_{\mathrm{Dk}}$ ranging from 20 to $40 \mathrm{~s}$ and support $\mathrm{T}_{\mathrm{DL}}$ ranging from 15 to $25 \mathrm{~s}$. Figure 12 depicts the values of the $C_{\text {ope }}$ that comply with possibility $\alpha_{\text {obj }}=0,7$ (and thus, necessity 0 ).

The operative capacity figure shows that, for any particular value of $T_{D k}$, a certain sensitivity in $T_{D L}$ can be observed. As a conclusion, in general the wider the $\mathrm{T}_{\mathrm{DL}}$ span, the higher the $\mathrm{C}_{\text {ope }}$ obtained. This happens because when the support of the fuzzy maximum capacity is bigger, the calculated $C_{\text {ope }}$ for $\alpha=0,7$ increases. Figure 13 shows this behavior in detail for a fixed value of $\mathrm{T}_{\mathrm{Dk}}(30 \mathrm{~s})$. The fuzzy capacity is depicted for three different values of $\mathrm{T}_{\mathrm{DL}}$.

That is, when the support of $\tilde{T}_{D}$ increases, lower values of dwell times are considered possible, and for these lower values of dwell times, higher values of maximum capacity are calculated as possible as well.

On the other hand, if a necessity value is imposed, the higher the $\mathrm{T}_{\mathrm{DL}}$, the lower the $\mathrm{C}_{\text {ope }}$ calculated (see Fig. 14). In this case, when the support of $\tilde{T}_{D}$ increases, higher values of dwell times are considered possible, and for these values of dwell times, lower values of maximum capacity are calculated as possible. Thus, when the target necessity level is imposed, the calculated $C_{\text {ope }}$ is lower as the $\tilde{\mathrm{C}}$ support increases.

Figure 15 shows the impact on $\mathrm{C}_{\mathrm{ope}}$ of modifying $\mathrm{T}_{\mathrm{Dk}}$ for different values of the necessity value imposed. This time $\mathrm{T}_{\mathrm{DL}}$ is fixed at $20 \mathrm{~s}$.

Then, by making use of this information and his own record of operating experience, the operator can design the timetable in order to maintain an occupancy time rate that allows certain reliability margins.

This may end up being a better solution than the one-size-fits-all approach of UIC's occupancy time rate guidelines. Also, by adjusting the possibility and

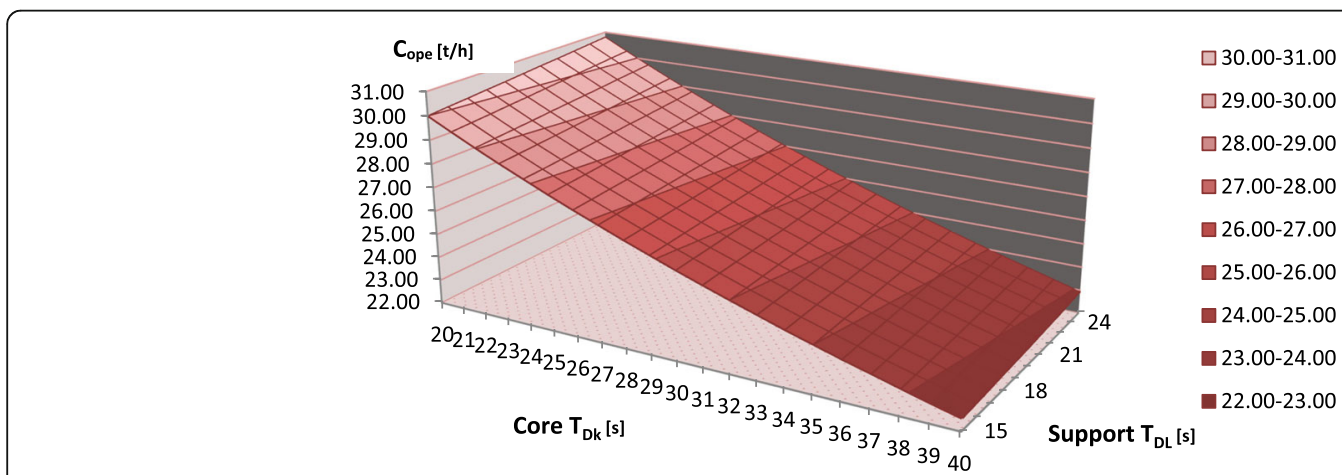

Fig. 12 Operative Capacity sensitivity analysis. Possibility $\left(a_{o b j}\right)=0,7$ Necessity $=0$ 


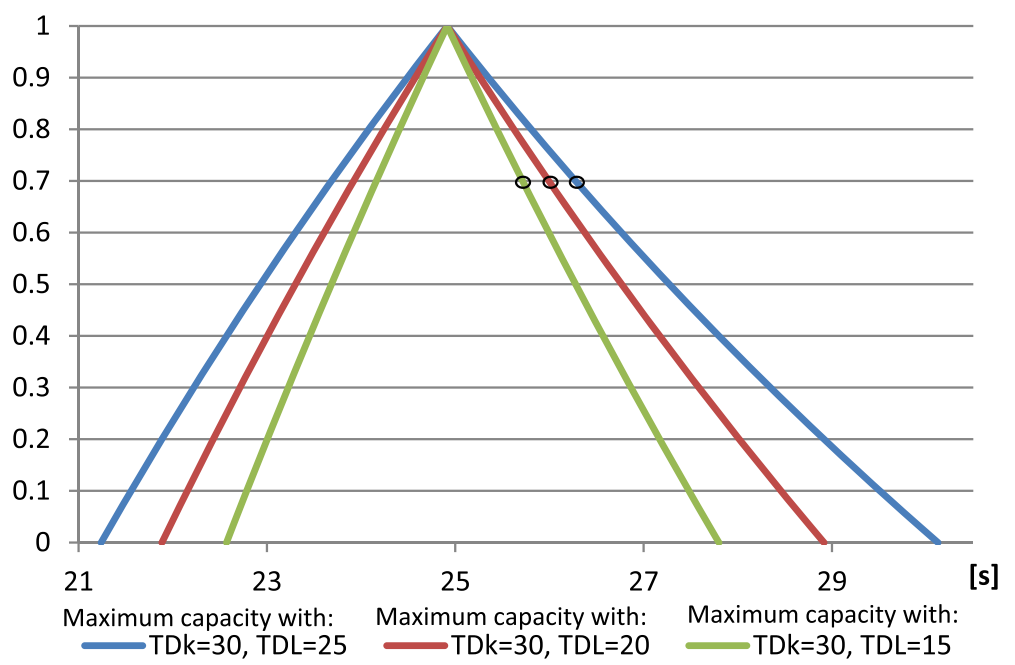

Fig. 13 Influence of $T_{D L}$ support variation on Fuzzy Maximum Capacity and $C_{o p e}$ (Possibility $=0.7$, Necessity $=0$ )

necessity target values, the system can be suited to account for a predefined level of uncertainty. The operator expertise again can be the key in its definition.

\section{Conclusions}

The main goal of this work is to improve urban railway lines capacity analysis considering the uncertainty associated to dwell times. In these type of lines dwell times have an important impact on capacity because there are frequent stops, and running times are quite stable due to the use of automatic driving systems.

New capacity measures have been proposed: the Fuzzy Maximum Capacity and the Fuzzy Occupancy Time Rate. They are based on the maximum capacity and occupancy time rates defined in [29] (timetable compression method), including the uncertainty associated with dwell times modeled as fuzzy numbers.

Three practical problems from the perspective of the traffic operator have been presented and solved applying the proposed model, by means of the alpha-cut arithmetic: (1) Is the operative capacity achievable?, (2) Does the operative capacity keep enough reliability margins?, (3) Which is the highest operative capacity that fulfills the recommended UIC Occupancy Time margins? Problems (1) and (2) are solved by calculating the degree of compliance in terms of possibility and necessity measures, while the third one calculates the maximum operative capacity that achieves the UIC robustness requirement with a given level of possibility or necessity as a target.

The proposed model has been applied to the section Gràcia-Sarrià of the Spanish railway operator FGC. The

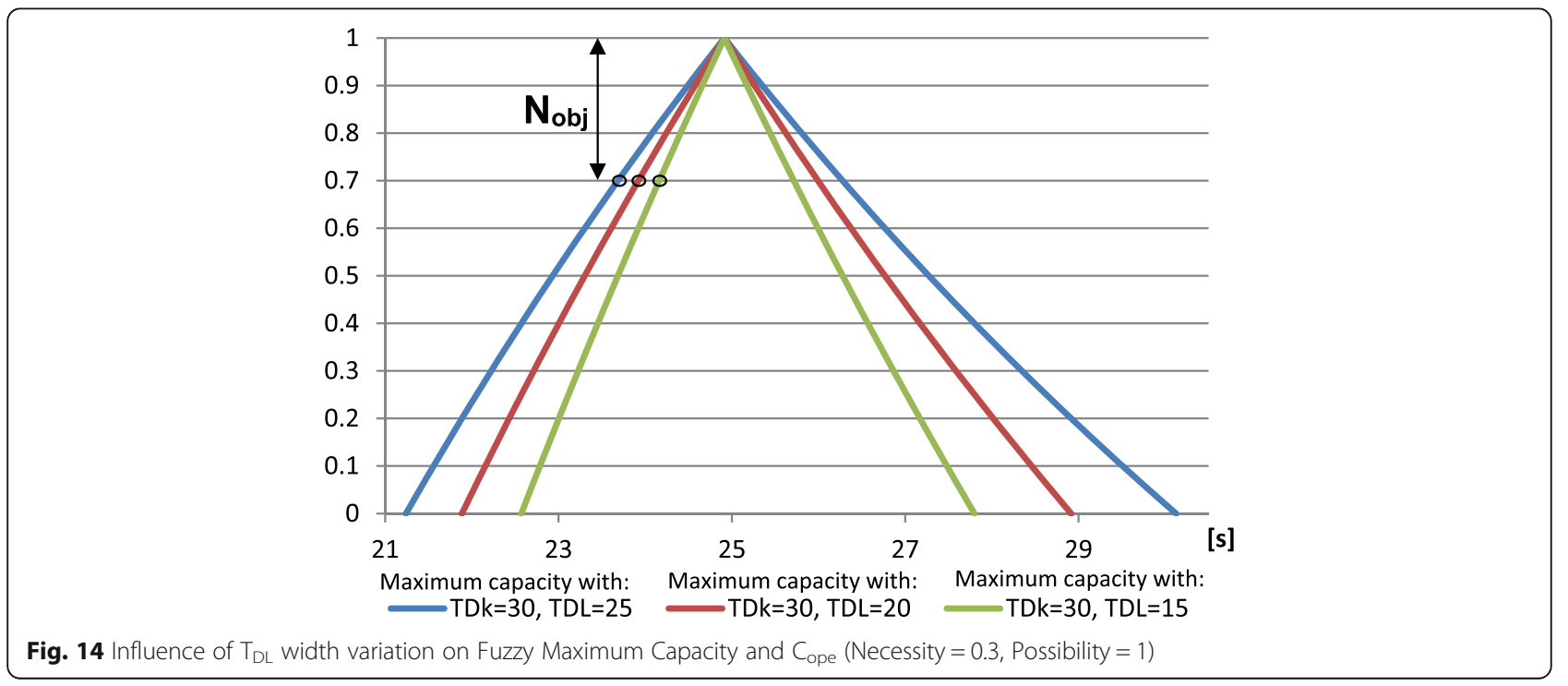




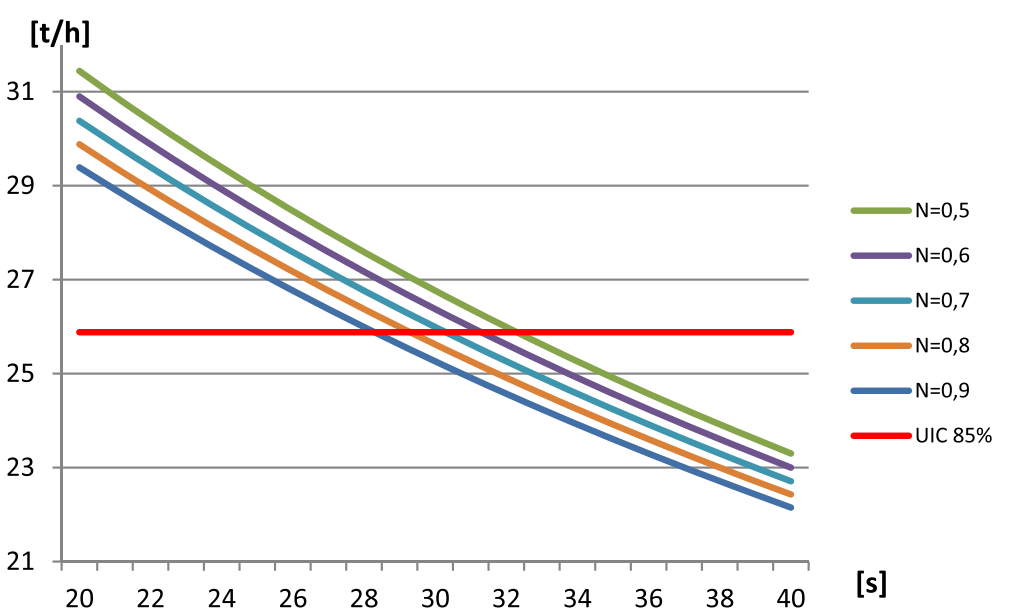

Fig. 15 Operative Capacity VS $T_{D k}$ for different values of Necessity. $T_{D L}=20 \mathrm{~s}$

model uses a railway simulator that enables the timetable compression method to obtain minimum conflict-free cycle times of the section under study.

It has been shown that the proposed method provides more information to the railway operator than the standard UIC method that does not include uncertainty regarding dwell times. The model permits as well adjusting the level of fulfillment of the UIC time margins to calculate the operative capacity. Furthermore, the sensitivity of the transport capacity to the uncertainty level for these input parameters of the model has been analyzed.

\section{Acknowledgements}

Not applicable.

\section{Funding}

No external funding was received for this project.

\section{Availability of data and materials}

The data input used for this project simulations belongs to FGC, a Spanish railway operator. Sharing that data could breach their privacy policies.

\section{Authors' contributions}

LMN carried out the simulations, study design and data analysis, also draft the full manuscript. AFC and APC participated in the overall proposal of the idea and also the design of the study and simulations. All authors read and approved the final manuscript.

\section{Authors' information}

Luis Miguel Navarro received the Industrial Engineering degree from the Universitat Politècnica de Catalunya, Terrassa, in 2007 and the Business Management degree from the Universitat Oberta de Catalunya, Barcelona, in 2014. He joined Siemens Rail Automation in 2010 and works as a certified Project Manager in Barcelona. He has been engaged in design and installation of signaling systems since 2010. His research interests include train simulation, railway capacity analysis and fuzzy systems.

Antonio Fernández-Cardador received the Physics degree from the Universidad Complutense de Madrid, Madrid, Spain, in 1991 and the Ph.D. degree from Comillas Pontifical University, Madrid, in 1997. He is a Research Fellow at the Railways Research Group, Institute for Research in Technology, and a Full Professor at ICAI School of Engineering, Comillas Pontifical University. His research interests include train simulation, railways operation and control, eco-driving, and railway capacity.
Asunción P. Cucala received the Electrical Engineering and Ph.D. degrees from Comillas Pontifical University, Madrid, Spain, in 1995 and 2003, respectively. She is a Research Fellow at the Railways Research Group, Institute for Research in Technology, and an Assistant Professor at ICAI School of Engineering, Comillas Pontifical University. Her research interests include energy efficiency in railways, railway capacity analysis, train simulation, and railways operation and control.

\section{Competing interests}

The authors declare that they have no competing interests.

\section{Publisher's Note}

Springer Nature remains neutral with regard to jurisdictional claims in published maps and institutional affiliations.

\section{Author details}

${ }^{1}$ Siemens Rail Automation S.A.U, Cornellà de Llobregat, Spain. ${ }^{2}$ Institute for research in technology, ICAI School of Engineering, Comillas Pontificial University, Madrid, Spain.

Received: 16 June 2018 Accepted: 21 November 2018

Published online: 22 December 2018

\section{References}

1. Abril M, Barber F, Ingolotti L, Salido MA, Tormos P, Lova A (2008) An assessment of railway capacity. Transport Res E-Log 44(5):774-806

2. Ballis a, Liberis K, Moschovou T (2004) Investigating the capacity of a metro line by means of a simulation model. Proc Inst Mech Eng F J Rail Rapid Transit 218(1):67-78

3. Chanas S, Kołodziejczyk W, Machaj A (1984) A fuzzy approach to the transportation problem. Fuzzy Sets Syst 13:211-221

4. Chu W-J, Zhang X-C, Chen J-H, Xu B (2015) An ELM-based approach for estimating train dwell time in urban rail traffic. Math Probl Eng 2015:473432

5. Cucala AP, Fernández A, Sicre C, Domínguez M (2012) Fuzzy optimal schedule of high speed train operation to minimize energy consumption with uncertain delays and drivers behavioral response. Eng Appl Artif Intell 25(8):1548-1557

6. Dicembre a, Ricci S (2011) Railway traffic on high density urban corridors: capacity, signalling and timetable. J Rail Transp Plann Manage 1(2):59-68

7. Domínguez M, Fernández A, Cucala AP, Lukaszewicz P (2011) Optimal design of metro automatic train operation speed profiles for reducing energy consumption. Proc Inst Mech Eng F J Rail Rapid Transit 225(5): 463-473

8. Dubois D, Prade H (1983) Ranking fuzzy numbers in the setting of possibility theory. Inf Sci 30(3):183-224

9. Fay A (2000) A fuzzy knowledge-based system for railway traffic control. Eng Appl Artif Intell 13(6):719-729 
10. Goverde RMP, Corman F, D'Ariano A (2013) Railway line capacity consumption of different railway signalling systems under scheduled and disturbed conditions. J Rail Transp Plann Manage 3(3):78-94

11. Hansen IA (2009) Railway network timetabling and dynamic traffic management. In: 2nd international conference on recent advances in railway engineering (ICRARE-2009) Iran university of science and technology Tehran I.R. Iran -Sep 27-28, 2009, Tehran, Iran, pp 135-145

12. Isaai MT, Kanani A, Tootoonchi M, Afzali HR (2011) Intelligent timetable evaluation using fuzzy AHP. Expert Syst Appl 38(4):3718-3723

13. Jiang Z, Xie C, Ji T, Zou X (2015) Dwell time modelling and optimized simulations for crowded rail transit lines based on train capacity. Promet Traffic\&Transportation 27(2):125-135

14. Kittelson \& Associates, Parsons Brinckerhoff, KFH Group, Texas A\&M Transportation Institute, \& Arup (2013) Transit capacity and quality of service manual. TCRB Rep 165:52

15. Kontaxi E, Ricci S (2010) Railway capacity analysis: methodological framework and harmonization perspectives. In: Proceedings of the 12th world conference on transportation research, Lisbon, pp 1-21

16. Krueger H (1999) Parametric modeling in rail capacity planning. In: WSC'99. 1999 winter simulation conference proceedings, vol 2, pp 1194-1200

17. Landex A (2012) Network effects in railways. WIT Trans Built Environ 127: 391-402

18. Medeossi G, Longo G, de Fabris S (2011) A method for using stochastic blocking times to improve timetable planning. J Rail Transp Plann Manage 1(1):1-13

19. Milinković S, Marković M, Vesković S, Ivić M, Pavlović N (2013) A fuzzy Petri net model to estimate train delays. Simul Model Pract Theory 33:144-157

20. Nash A, Huerlimann D (2004) Railroad simulation using OpenTrack. Comput Railw IX:45-54

21. Ortega Riejos F, a, Barrena E, Canca Ortiz JD, Laporte G (2015) Analyzing the theoretical capacity of railway networks with a radial-backbone topology. Transp Res A Policy Pract. https://doi.org/10.1016/j.tra.2015.03.018

22. Perkins A, Ryan B, Siebers $P$ (2014) Modelling and simulation of rail passengers to evaluate methods to reduce dwell times. In: 14th international conference on modeling and applied simulation, pp 132-141

23. Robert L, Burdett RL, Kozan E (2006) Techniques for absolute capacity determination in railways. Trans Res B Methodological 40(8):616-632. Retrieved from http://eprints.qut.edu.au/10385/1/10385.pdf

24. Salido MA, Tarazona S (n.d.) Robustness in railway timetables. In 7th World Congress on Intelligent Control and Automation, 2008, Chongqing, China

25. San HP, Masirin MIM (2016) Train dwell time models for rail passenger service. In: MATEC Web of Conferences, vol 47, pp 3005-1-3005-6

26. UIC (1996) Leaflet 405 OR- Links between Railway Infrastructure Capacity and the Quality of Operations, 1st edn

27. UIC (2008). Influence of ETCS on line capacity - Generic study

28. UIC (2010). Influence of the European Train Control System (ETCS) on the capacity of nodes

29. UIC (2013) Code 406 Capacity, 2nd edn

30. United Nations, Department for Economic and Social Affairs, P. D. (2015) World Urbanisation Prospects: The 2014 Revision

31. Yang L, Li K, Gao Z (2009) Train timetable problem on a single-line railway with fuzzy passenger demand. IEEE Trans Fuzzy Syst 17(3):617-629

32. Zhang Q, Han B, Li D (2008) Modeling and simulation of passenger alighting and boarding movement in Beijing metro stations. Transp Res C Emerg Technol 16(5):635-649

\section{Submit your manuscript to a SpringerOpen ${ }^{\circ}$ journal and benefit from:}

- Convenient online submission

- Rigorous peer review

- Open access: articles freely available online

- High visibility within the field

- Retaining the copyright to your article

Submit your next manuscript at $\boldsymbol{\nabla}$ springeropen.com 